University of Wollongong

Research Online

Faculty of Engineering and Information

Faculty of Engineering and Information

Sciences - Papers: Part A

Sciences

$1-1-2016$

\title{
A magnetically actuated drug delivery system for robotic endoscopic capsules
}

Fredy Munoz

University of Wollongong, ffm517@uowmail.edu.au

Gursel Alici

University of Wollongong, gursel@uow.edu.au

Weihua Li

University of Wollongong, weihuali@uow.edu.au

Follow this and additional works at: https://ro.uow.edu.au/eispapers

Part of the Engineering Commons, and the Science and Technology Studies Commons

Research Online is the open access institutional repository for the University of Wollongong. For further information contact the UOW Library: research-pubs@uow.edu.au 


\title{
A magnetically actuated drug delivery system for robotic endoscopic capsules
}

\begin{abstract}
There is an increasing need to incorporate an actively controlled drug delivery system (DDS) into the next generation of capsule endoscopy in order to treat diseases in the gastrointestinal tract in a noninvasive way. Despite a number of attempts to magnetically actuate drug delivery mechanisms embedded in endoscopic capsules, longer operating distances and further miniaturization of on-board components are still drawbacks of such systems. In this paper, we propose an innovative magnetic system that consists of an array of magnets, which activates a DDS, based on an overly miniaturized slider-crank mechanism. We use analytical models to compare the magnetic fields generated by cylindrical and arc-shaped magnets. Our experimental results, which are in agreement with the analytical results, show that an optimally configured array of the magnets enhances the magnetic field and also the driving magnetic torque and subsequently, it imposes a high enough force on the piston of the DDS to expel a required dose of a drug out of a reservoir. We conclude that the proposed magnetic field optimization method is effective in establishing an active DDS that is designed to deliver drug profiles with accurate control of the release rate, release amount, and number of doses.
\end{abstract}

\section{Keywords}

drug, delivery, system, robotic, endoscopic, capsules, actuated, magnetically

Disciplines

Engineering | Science and Technology Studies

\section{Publication Details}

Munoz, F., Alici, G. \& Li, W. (2016). A magnetically actuated drug delivery system for robotic endoscopic capsules. Journal of Medical Devices, Transactions of the ASME, 10 (1), 11004-1-11004-11. 


\section{Fredy Munoz \\ School of Mechanical, Materials and Mechatronic Engineering, \\ University of Wollongong, \\ Wollongong, NSW 2522, Australia \\ e-mail: ffm517@uowmail.edu.au \\ A Magnetically Actuated Drug \\ Delivery System for Robotic Endoscopic Capsules}

Gursel Alici ${ }^{1}$

ARC Center of Excellence for Electromaterials Science,

School of Mechanical, Materials and

Mechatronic Engineering,

University of Wollongong,

Wollongong, NSW 2522, Australia

e-mail: gursel@uow.edu.au

Weihua Li

School of Mechanical, Materials and Mechatronic Engineering, University of Wollongong,

Wollongong, NSW 2522, Australia

e-mail: weihuali@uow.edu.au

There is an increasing need to incorporate an actively controlled drug delivery system $(D D S)$ into the next generation of capsule endoscopy in order to treat diseases in the gastrointestinal tract in a noninvasive way. Despite a number of attempts to magnetically actuate drug delivery mechanisms embedded in endoscopic capsules, longer operating distances and further miniaturization of on-board components are still drawbacks of such systems. In this paper, we propose an innovative magnetic system that consists of an array of magnets, which activates a DDS, based on an overly miniaturized slider-crank mechanism. We use analytical models to compare the magnetic fields generated by cylindrical and arc-shaped magnets. Our experimental results, which are in agreement with the analytical results, show that an optimally configured array of the magnets enhances the magnetic field and also the driving magnetic torque and subsequently, it imposes a high enough force on the piston of the DDS to expel a required dose of a drug out of a reservoir. We conclude that the proposed magnetic field optimization method is effective in establishing an active DDS that is designed to deliver drug profiles with accurate control of the release rate, release amount, and number of doses. [DOI: 10.1115/1.4031811]

\section{Introduction}

A DDS is an important feature to be added to the next generation of wireless capsule endoscopes (WCE) not only to treat diseases in the digestive system, such as obscure gastrointestinal bleeding, Crohn's disease, small bowel tumors, and celiac disease, but also to conduct drug absorption studies. Different approaches have been proposed in the last two decades to design DDS for WCE which include anchoring and active drug release mechanisms. An active drug release mechanism that enables the generation of different drug profiles (i.e., accurate control of release rate, release amount, and number of doses) is desirable for an efficient on-demand DDS for WCE [1]. Only two studies have reported promising results for an on-demand DDS for WCE [2,3]. The former [2] used a battery and microelectronic components in the prototype of a CE and the latter [3] used a magnetic field. Although the use of batteries and microelectronic components reduces the difficulties associated with the operation of an actuation system inside the WCE, the small volume of a WCE imposes limitations on the drug chamber size and the number of components to be placed in a DDS module [1]. Unless all components of a WCE can be further miniaturized with the existing technology, this approach does not seem to be practical. On the other hand, magnetic systems have been used in different medical applications since magnetic systems are considered safe for biological tissues and cells and can potentially be scaled down to actuate overly miniaturized systems [4-9].

The remote actuation of a variety of mechanisms in prototypes of WCE has been achieved by means of magnetic systems. For example, cylindrical magnets have been used to control the trajectory of a CE [10], to deploy legs to provide the locomotion of a CE $[11,12]$, to perform biopsy procedures $[13,14]$, and also to achieve wireless insufflation [15]. In addition, cylindrical magnets have been also used to release a drug from a chamber in a prototype of WCE [3]. Although the results reported in these studies suggest that magnetic systems can potentially be used to remotely

${ }^{1}$ Corresponding author.

Manuscript received June 20, 2015; final manuscript received September 30 , 2015; published online November 16, 2015. Assoc. Editor: Rupak K. Banerjee. control a drug release mechanism embedded in a WCE, at least two problems must be solved in order to use such magnetic propulsion systems. The first one is related to the operating distance and the second one is the miniaturization of the on-board permanent magnets placed inside the capsule.

In all those studies [3,10-15], the maximum operating distance is $150 \mathrm{~mm}$ which is not large enough for a realistic WCE application. In order to overcome this problem, we propose a magnetomechanical system made of an array of multiple permanent magnets, which enhances the magnetic field when the operating distance is increased to a more realistic distance of $240 \mathrm{~mm}$. We have shown that the miniaturization of an active drug delivery mechanism in the WCE is possible when the external magnetic field is properly applied. In this work, we report on the design optimization of a magnetic system which can be realized by an array of magnets. This design allows large operating distances and does not impose strict design constraints on the miniaturization of the components inside a WCE. We reported preliminary numerical results for a magnetomechanical system for DDS in WCE in Ref. [16]. The primary contribution of this study is to present the synthesis of an optimized DDS based on a miniaturized slider-crank mechanism articulated with an optimized magnetic torque. This innovative DDS is designed and implemented by comparing analytical and experimental results for the magnetic flux density, the magnetic torque, and the force transmitted to the drug delivery chamber (i.e., piston) while minimizing the size of the magnet to be embedded in the WCE and maximizing the distance from the center of the WCE to the center of the external magnetic source. Specifically, we present an overview of this medical application and set the limitations of this study in Sec. 2. We continue our analysis and compare the source of the magnetic field created by the arrays of cylindrical and arc-shaped magnets in Sec. 3. Section 4 describes the details of the experimental setup to verify the concept of an array of multiple magnets to provide an optimized magnetic field.

A comparison between the analytical and experimental results for an array of arc-shaped magnets and the actuation of a slider-crank mechanism and drug delivery capability of the mechanism are presented in Sec. 5. Finally, a discussion on the results and future work is presented in Sec. 6. 


\section{DDS Overview}

2.1 Overall Medical Application. The entire DDS for WCE based on magnetic actuation is shown in Fig. 1. This system consists of three main components: the external magnetic system made of permanent magnets (A), which surrounds the patient, the drug release module (B) embedded in the robotic capsule (C), and three complementary modules (D) integrated in the robotic capsule. The components of the drug release module are: an internal permanent magnet (IPM), a slider crank mechanism that is connected to the IPM, a drug reservoir to store the drug to be released, and an orifice through which the drug is expelled.

Figure 2 shows the details of the components embedded in the robotic capsule that would allow the release of drug from the drug reservoir. The slider crank mechanism consists of a piston (B) that is linearly moved by two connecting rods $(C)$ that are attached to two rotating disks (D). The rods have holes on both ends. One end of the rod is inserted in a piston slot and its other end is connected to a pin on a disk. One disk is placed at the top of the IPM (A) and another disk is placed at its bottom side. These disks rotate about the crankshaft when the IPM is driven by the magnetic torque $\tau_{z}^{c}$. The crankshaft that is connected to the IPM is also inserted in the

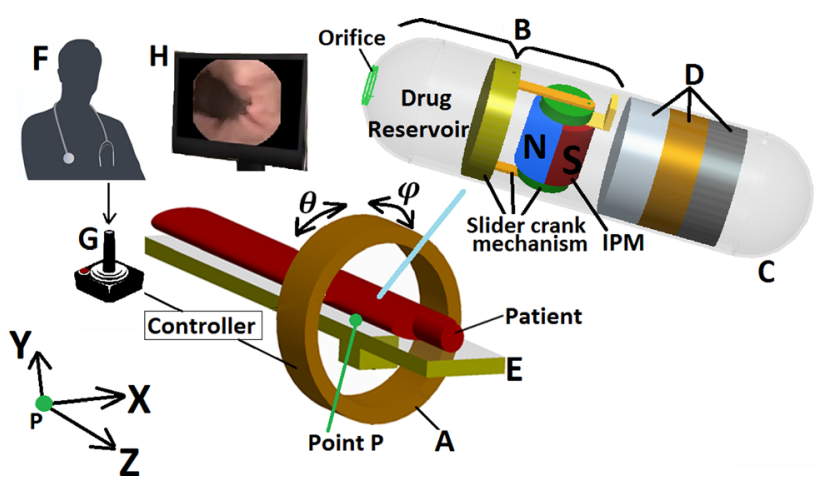

Fig. 1 The main components of the proposed DDS for WCE. A: ring-shaped external magnetic system, B: drug release module, $\mathrm{C}$ : the robotic capsule, D: complementary modules within the capsule (anchoring mechanism, active locomotion system, and localization and orientation detection module), E: patient bed, F: clinician, G: joystick, and H: human capsule interface. Point $P$ represents the origin of the general coordinate system $X Y Z, \theta$ is taken with respect to the $x$-axis, and $\varphi$ is taken with respect to the $z$-axis.
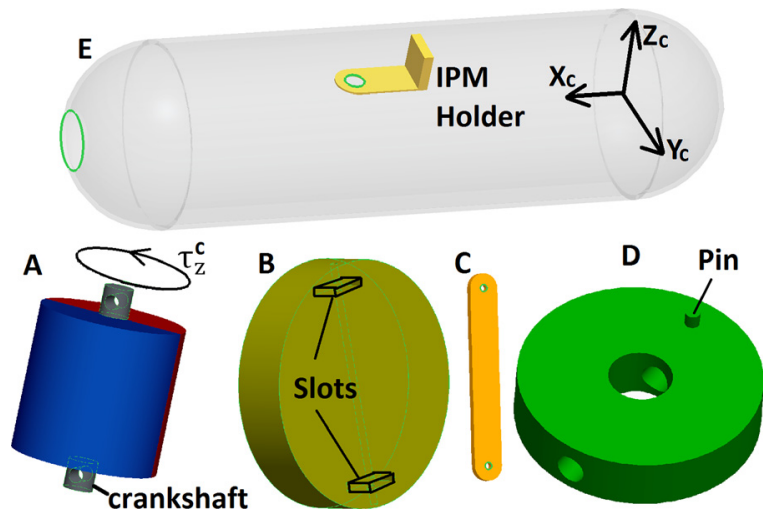

Fig. 2 The components of the slider crank mechanism. A: IPM, $\mathrm{B}$ : disk-shaped piston, C: connecting rod, $\mathrm{D}$ : disk, and $\mathrm{E}$ : robotic capsule. The coordinate system $X_{c} Y_{c} Z_{c}$ of the IPM with respect to the general reference system $X Y Z$ is shown in Fig. 1. hole in the IPM holder. The IPM holder is fixed and attached to the internal wall of the robotic capsule (E).

The total volume of a swallowable capsule is estimated to be at most $3 \mathrm{ml}(11 \mathrm{~mm}$ in diameter and $26 \mathrm{~mm}$ long $)$ and a drug reservoir volume between $0.17 \mathrm{ml}$ and $1 \mathrm{ml}$ seems to be a reasonable volume capacity for DDS in WCE [1,17]. Due to these size restrictions, we propose a dedicated robotic capsule for DDS. In other words, this robotic capsule does not include the image guidance module (e.g., battery, camera, and communication capabilities) to perform the screening procedures that are currently achieved with commercial endoscopic capsules. Therefore, our proposed robotic capsule only possesses those specific modules that are relevant to successfully achieve drug delivery (i.e., the three modules (D) shown in Fig. 1 along with the drug release module (B)) and we aim to create a drug reservoir volume of at least $0.5 \mathrm{ml}$. Nevertheless, the inclusion of an image guidance module in our proposed robotic capsule would make the drug release procedure more accurate. The necessity to include multiple on-board modules in the robotic capsule again emphasizes the requirement of miniaturizing the active drug release mechanism as much as possible.

Regarding the anchoring module in the capsule, its anchoring force should be approximately $912 \mathrm{mN}$ [17]. This magnitude would exert a pressure of $1 \mathrm{kPa}$ on the intestine wall if we consider the contact area of a commercial WCE. This pressure is below the pain level of $5 \mathrm{kPa}$ [3]. The piston force of our drug release module must be lower than the anchoring force. Otherwise, the capsule may detach from the intestine wall. Therefore, a peak piston force of $820 \mathrm{mN}$ that is below the pain level would be sufficient to release different drug compounds [16]. A last consideration is related to the magnitude of the magnetic field created by the external magnetic system. In this work, we use a safety threshold of $2 \mathrm{~T}$ recommended for occupational exposure [13].

2.2 Clinical Procedure. The proposed clinical procedure is as follows. After the patient's digestive system is screened and anomalies are detected, the patient would undergo a therapeutic procedure that may include the delivery of drugs at target regions within the digestive system. In this case, the patient lies in a bed and swallows a new robotic capsule that includes a drug release module and the three complementary on-board modules shown in Fig. 1-D. Then, the doctor drives the robotic capsule to the target area by controlling its position remotely. To do this, the doctor activates the locomotion system embedded in the capsule while the external magnetic system, the position and orientation of which can be controlled by a joystick, is placed at an appropriate distance from the patient where it transmits no torque on the IPM, thus preventing the activation of the drug release module during this phase. The localization and orientation module within the capsule wirelessly transmits the capsule's position in real time to a human machine interface. Once the capsule reaches the target area, the doctor activates remotely the anchoring mechanism within the capsule to make sure it is firmly fixed on the intestine's surface. The activation and deactivation of these three complementary modules must be compatible with the magnetically actuated DDS.

After the robotic capsule is properly anchored, the doctor uses the joystick to place the external magnetic system in the correct position and with the correct orientation to activate the drug release module (i.e., to impart a magnetic torque $\tau_{z}^{c}$ to the IPM). This activation can be achieved by following the next two sequential steps: first, a coordinate system $X_{\mathrm{a}} Y_{\mathrm{a}} Z_{\mathrm{a}}$, that is associated with the external magnetic system, is adjusted with respect to the general reference system $X Y Z$ shown in Fig. 1. Second, the external magnetic system, which can be powered by motors, starts rotating about its $Z_{\mathrm{a}}$ axis, generating in this way a rotating magnetic field that can impart a magnetic torque $\tau_{z}^{c}$ to the IPM. The rotation of the IPM about its axial axis $Z_{\mathrm{c}}$ is converted into a linear movement by the slider crank mechanism and the piston pushes the drug out 
of the reservoir. By controlling the external magnetic system's rotational speed and direction (clockwise or counterclockwise), the doctor is able to control the release rate, release amount, and number of doses. These are highly desirable variables to be controlled in an on-demand DDS for WCE to produce different drug profiles [1]. Finally, after the drug is released, the doctor deactivates the anchoring mechanism and reactivates the locomotion module to propel the robotic capsule.

2.3 Objectives and Limitations. Our proposed DDS for WCE uses different mechanisms and modules (see Fig. 1) to achieve on-demand drug release. Although each module and component could be optimized to improve the overall system, in this paper we mainly focus on the optimization of the magnetic interactions between the external magnetic system that is made of external permanent magnets (EPMs) and the IPM. Therefore, some considerations regarding the spatial position and orientation of these permanent magnets are presented as follows.

In the real application, the robotic capsule is free to move and rotate within the cylindrical volume of radius $d$ and length $L$ of the external magnetic system. However, in order to facilitate the analysis of the magnetic interactions between the EPMs of the external magnetic system and the IPM, we introduce the following specific physical constraints on these permanent magnets. First, we align the two coordinate systems $X Y Z$ and $X_{\mathrm{a}} Y_{\mathrm{a}} Z_{\mathrm{a}}$ by coinciding the center of the external magnetic system with point $P$ and by putting in parallel planes $X Y$ and $X_{\mathrm{a}} Y_{\mathrm{a}}$ (i.e., the external magnetic system is not inclined, thus $\varphi=0 \mathrm{deg}$ ). Therefore, the ring-shaped external magnetic system can only rotate about the $Z$-axis (i.e., $0 \mathrm{deg} \leq \theta<360 \mathrm{deg}$ ). Nevertheless, in the real application, the external magnetic system's location and orientation could be controlled by adjusting its center and its angles $\theta$ and $\varphi$ with the joystick and a transformation matrix can be easily used when working with $X Y Z$ and $X_{\mathrm{a}} Y_{\mathrm{a}} Z_{\mathrm{a}}$ reference systems. Second, although the IPM's axial axis $\left(Z_{c}\right)$ can also be inclined with respect to the $Z_{\mathrm{a}}$ axis of the external magnetic system, we constrain it in this paper to be always parallel to $Z_{\mathrm{a}}$. The analysis of the transmitted torque $\tau_{z}^{c}$ when the IPM's axial axis is tilted is then deferred to a future work. Third, we also make the plane $X_{\mathrm{c}} Y_{\mathrm{c}}$ of the IPM coincide with the plane $X_{\mathrm{a}} Y_{\mathrm{a}}$ of the external magnetic system, so that axial movements of the IPM are not part of this study.

Finally, since the ring-shaped external magnetic system is symmetrical in the $z$-axis, we choose half of the length $L$ to be the plane $z=0$ as the plane on which the IPM's center moves within a circle of radius $d$ centered at point $P$. With these constraints, we only allow the movement of the IPM's center on the plane $z=0$ with the IPM's axial axis parallel to the $Z$-axis, and subsequently $\tau_{z}^{c}$ can be simply denoted as $\tau_{z}$. Keeping in mind that the magnetic flux density created by the EPMs, $B_{\text {ext }}$, decreases with the distance and point $P$ is located at the furthest distance from the inner surface of the external magnetic system (on plane $z=0$ ), then point $P$ represents a critical point for $B_{\text {ext }}$ and also for $\tau_{z}$. Therefore, we aim to increase $B_{\text {ext }}$ at point $P$ and also the magnetic torque imparted on the IPM, the center of which is located at that critical point $P$. Higher $B_{\text {ext }}$ and magnetic torques are then expected at any other point within the circle with a radius of $d$, where the IPM's center can be placed. If the IPM's center moved axially or radially away from point $P$, then the position and orientation of the external magnetic system can be controlled from the joystick to obtain an adequate alignment between the EPMs and the IPM.

Finding an optimal configuration of EPMs within the ring of the external magnetic system is crucial to improve the magnetic torque that is transmitted to the IPM, and this may allow the miniaturization of the IPM's size and increase the operating distance of the DDS at the same time. Therefore, we present, in Sec. 3, the optimization of the EPMs to increase $B_{\text {ext }}$ at point $P$. This process is carried out using analytical models.

\section{Magnetic Field Analysis}

3.1 Magnetic Systems Consisting of Arrays of Multiple Permanent Magnets. The magnetic torque $\tau_{z}$ exerted on the IPM with a volume $V$ and magnetization $m$ is given by $[16,18]$

$$
\tau_{z}=\frac{V}{\mu_{0}} *|\mathbf{m}| *\left|\mathbf{B}_{\text {ext }}\right| * \sin \left(\varnothing_{\mathrm{m}}\right) \quad(\mathrm{N} \cdot \mathrm{m})
$$

where $\mu_{0}$ is the permeability of the free space (i.e., 4 $\pi \times 10^{-7} \mathrm{H} / \mathrm{m}$ ), and the units for magnetization and magnetic flux density are Tesla. $\varnothing_{\mathrm{m}}$ represents the misalignment angle between $\mathbf{m}$ and $\mathbf{B}_{\text {ext }}$. The torque $\tau_{z}$ will tend to orient the vector $\mathbf{m}$ along $\mathbf{B}_{\text {ext }}$ and may generate a rotational movement about the $\mathrm{IPM}^{\prime}$ s rotational axis. Since the magnetic torque is proportional to $\left|\mathbf{B}_{\text {ext }}\right|$, we aim to enhance $\left|\mathbf{B}_{\text {ext }}\right|$ at the critical point $P$. It should be noted that only $B_{x}$ and $B_{y}$ components of $\mathbf{B}_{\text {ext }}$ contribute to $\tau_{z}$. Although the magnetic torque is also proportional to the IPM's volume $V$, variations in the IPM's dimensions and their effects on the magnetic torque and piston force will be presented in the experimental section (i.e., Sec. 5.3).

In our previous work [16], we used a finite-element solution (i.e., Comsol) and showed that multiple EPMs can be used to create a stronger $\left|\mathbf{B}_{\text {ext }}\right|$ than the one produced by a single EPM at point $P$. We also presented in our previous work that multiple EPMs (up to 8) arranged along a circle at appropriate locations and with certain orientations can impart higher magnetic torques to a small IPM. In this paper, we perform parametric studies to determine a suitable array of permanent magnets to be placed in the ring-shaped external magnetic system shown in Fig. 1 by using analytical models since these studies are extremely timeconsuming with finite-element methods [19].

The first type of array consists of diametrically magnetized cylindrical magnets and the second type consists of arc-shaped magnets, as shown in Fig. 3. Cylindrical magnets are commonly used in medical applications [10-15]. Arc-shaped magnets have shown promising results in the transmission of high density torques [20,21]. Therefore, we consider them in this study.

In order to understand the magnetic flux density produced by these arrays of permanent magnets, we analyze the contribution of each magnet, $i$, and then use the superposition principle to obtain the total magnetic flux density $\mathbf{B}_{\text {ext }}$.

By using the magnetic charge model [22], the magnetic flux density produced by an EPM of volume $V_{i}$ and magnetization $\mathbf{M}_{\mathbf{i}}\left(\mathbf{x}^{\prime}\right)$ is calculated as

$$
B_{\mathrm{ext}}^{i}(\mathbf{x})=\frac{1}{4 \pi} \int_{v i} \frac{\rho_{M i}\left(\mathbf{x}^{\prime}\right)\left(\mathbf{x}-\mathbf{x}^{\prime}\right)}{\left|\mathbf{x}-\mathbf{x}^{\prime}\right|^{3}} \mathrm{dVi}^{\prime}+\frac{1}{4 \pi} \int_{s i} \frac{\sigma_{M i}\left(\mathbf{x}^{\prime}\right)\left(\mathbf{x}-\mathbf{x}^{\prime}\right)}{\left|\mathbf{x}-\mathbf{x}^{\prime}\right|^{3}} \mathrm{dsi}^{\prime}
$$

where $\mathbf{x}$ is the observation point, $\mathbf{x}^{\prime}$ is the source point, and the integration is over the volume for which the magnetization exists. $s_{i}$ defines the surface that bounds $V_{i}$ (the surface of the $i$ th magnet

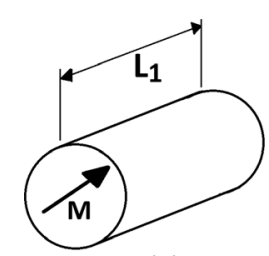

(a)

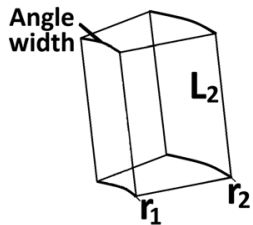

(b)

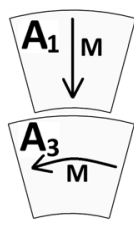

(c)
Fig. 3 (a) A diametrically magnetized cylindrical magnet with radius $R$, length $L_{1}=z_{2}-z_{1}$, and magnetization grade $M,(b)$ arc-shaped permanent magnet, and (c) top view of different types of arc-shaped permanent magnets (i.e., $A_{1}, A_{2}, A_{3}$, and $A_{4}$ ) used in this work 
that generates $\mathbf{B}_{\mathrm{ext}}^{\mathbf{i}}(\mathbf{x}) \cdot \rho_{M i}$ and $\sigma_{M i}$ are the volume and surface charge densities, respectively, and are given by

$$
\begin{array}{r}
\rho_{M i}\left(\mathbf{x}^{\prime}\right)=-\nabla^{\prime} \cdot \mathbf{M}_{\mathbf{i}}\left(\mathbf{x}^{\prime}\right) \\
\sigma_{M i}\left(\mathbf{x}^{\prime}\right)=\mathbf{M}_{\mathbf{i}}\left(\mathbf{x}^{\prime}\right) \cdot \hat{\mathbf{n}}
\end{array}
$$

where $\hat{\mathbf{n}}$ is the normalized vector perpendicular to the surface of the magnet, $i=1,2,3$, and 4 , and $\nabla^{\prime}$ operates on the primed coordinates. Equations (2)-(4) are general and can be used for any shape and size of magnets. We take $\mathbf{B}_{\text {ext }}(\mathbf{x})$ as the total flux density generated by each type of array of permanent magnets. In particular, $\left|\mathbf{B}_{\text {ext }}(\mathbf{x})\right|=\sqrt{B_{x}^{2}+B_{y}^{2}+B_{z}^{2}}$, but only the components of the magnetic field in the $x y$ plane will contribute to the magnetic torque about the $z$-axis, which is the axis of rotation of the IPM. We use the analytical models for $B_{\text {ext }}^{i}(\mathbf{x})$ generated by a diametrically magnetized cylindrical magnet [23] and by arc-shaped magnets [24] and also the superposition principle to find the total magnetic field generated by the arrays of magnets.

3.2 Comparison of the Magnetic Flux Density Created by Cylindrical and Arc-Shaped Magnets. In the analysis of the magnetic flux density created by the external magnetic system, we align $X_{\mathrm{a}}$ and $X$ axes (i.e., $\theta=0 \mathrm{deg}$ ) because there is no need to rotate the external magnetic system. We start by placing one cylindrical EPM (radius $R$ and length $L$, diametrically magnetized and magnetization grade $M$ ) at $\theta=180 \mathrm{deg}$ with its center located at a radial distance of $d+R$ from the center of the system (point $P)$ as shown in Figs. $4(a)$ and $4(b)$. We set a relatively large operating distance $d$ of $240 \mathrm{~mm}$ and align $\mathbf{M}$ with the $X$-axis to facilitate the analysis so that $\left|\mathbf{B}_{\text {ext }}(\mathbf{x})\right|=\sqrt{B_{x}^{2}+B_{y}^{2}}=B_{x}$ at point $P$. We aim to create a $B_{x}$ of $103 \mathrm{mT}$ at point $P$ since this value seems to be reasonable for the actuation of small IPMs [13].

We perform a parametric study using analytical models by varying $L$ and $R$ to find the minimum volume, $V_{\min }$, of the cylindrical EPM that creates $103 \mathrm{mT}$ from an operating distance $d$ of $240 \mathrm{~mm}$. Figures $4(c)$ and $4(d)$ show that although there are many ways to create $103 \mathrm{mT}$ from that distance (for example, a cylinder with $L=240 \mathrm{~mm}$ and volume of approximately $5.7 \times 10^{-2} \mathrm{~m}^{3}$ creates $103 \mathrm{mT}$, but also a cylinder with $L=600 \mathrm{~mm}$ and volume of approximately $4.5 \times 10^{-2} \mathrm{~m}^{3}$ creates $103 \mathrm{mT}$ ), there is a minimum cylindrical volume that generates $103 \mathrm{mT}$ from the distance $d$. We find the following optimal parameters for a cylindrical EPM diametrically magnetized: $R_{\text {optimal }}=175 \mathrm{~mm}$, $L_{\text {optimal }}=425 \mathrm{~mm}$, and $V_{\min }=40.9 \times 10^{-3} \mathrm{~m}^{3}$.

We replace this optimal cylindrical EPM with four cylindrical EPMs, each denoted as $C_{i}(i=1,2,3$, and 4$)$ and with $25 \%$ of the volume $V_{\min }$ (i.e., $10.225 \times 10^{-3} \mathrm{~m}^{3}, R=87.5 \mathrm{~mm}$, and $\left.L_{1}=425 \mathrm{~mm}\right)$ and place $C_{1}$ at $\theta=180 \mathrm{deg}, C_{2}$ at $\theta=0 \mathrm{deg}, C_{3}$ at $\theta=90 \mathrm{deg}$, and $C_{4}$ at $\theta=270 \mathrm{deg}$ with magnetization directions as shown in Fig. 5(a). We denote this configuration as $C_{1234} . B_{x}$ created by $C_{1234}$ at the center of the system is increased to $141 \mathrm{mT}$. Instead of using four cylindrical EPMs, if we use four arc-shaped magnets each with a volume $V_{\mathrm{A}}=V_{\min } / 4$, as shown in Fig. 5(b), then $B_{x}$ at the center of the system will be $158 \mathrm{mT}$. The specifications of each arc-shaped magnet, which in this configuration we denote as $A_{1234}$, are: $r_{1}=240 \mathrm{~mm}, r_{2}=386.6 \mathrm{~mm}$, $L_{2}=425 \mathrm{~mm}$, angular width $\Delta \theta$ of $30 \mathrm{deg}$, two segments radially magnetized (i.e., $A_{1}$ and $A_{2}$ ) and two other segments tangentially magnetized (i.e., $A_{3}$ and $A_{4}$ ). Magnetization grade: $1.32 \mathrm{~T}$. We also use analytical models and find that the positions of the arc-shaped magnets presented in Fig. 5(b) are optimal to increase $B_{x}$ at the center of the system and the details of this optimization process are presented in Ref. [25]. The same optimal configuration with four cubic magnets was reported in Ref. [26].

This indicates that given the distance $d$ and the magnitude of $B_{x}$ that we want to generate, it is more efficient to distribute the volume of the EPMs along the circle with radius $d$ than to allocate the entire optimal volume $V_{\min }$ to a single cylindrical EPM. Figure 6 compares $B_{x}$ along the $x$-axis for one optimized cylindrical EPM (with optimized volume $40.9 \times 10^{-3} \mathrm{~m}^{3}$ ), four cylindrical EPMs (each with a volume of $10.225 \times 10^{-3} \mathrm{~m}^{3}$ ), and four arc-shaped magnets (each with a volume of $10.225 \times 10^{-3}$ $\mathrm{m}^{3}$ ). The optimization of location, orientation, and shape of each EPM is of great importance when the magnetic system is scaledup since a minimum weight of the EPMs would be highly desirable not only to ease its maneuverability but also to reduce the costs associated with the fabrication of the EPMs.

Since for the same minimum volume $V_{\min }$, an array of four arcshaped magnets produces the highest magnetic field at the center of the system where the IPM is located, we conduct experiments

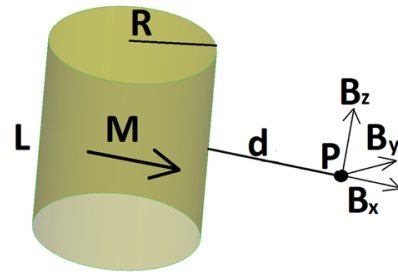

(a)

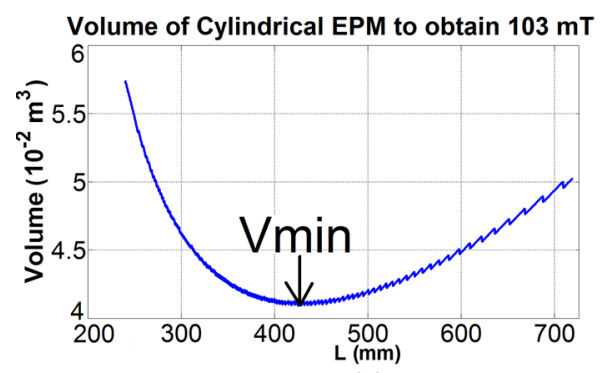

(c)

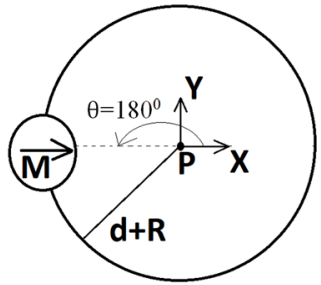

(b)

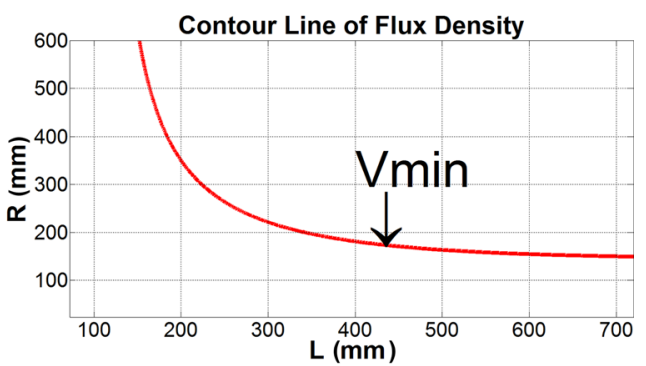

(d)

Fig. 4 (a) Single cylindrical EPM, (b) top view of the position and orientation of the EPM with respect to general reference system, (c) EPM's volume that produces $103 \mathrm{mT}$ from a distance $d=240 \mathrm{~mm}$, magnetization $M=1.32 \mathrm{~T}$, and $(d)$ contour line for $B_{x}=103 \mathrm{mT}\left(V_{\min }\right.$ occurs at $L=425 \mathrm{~mm}$ ) 


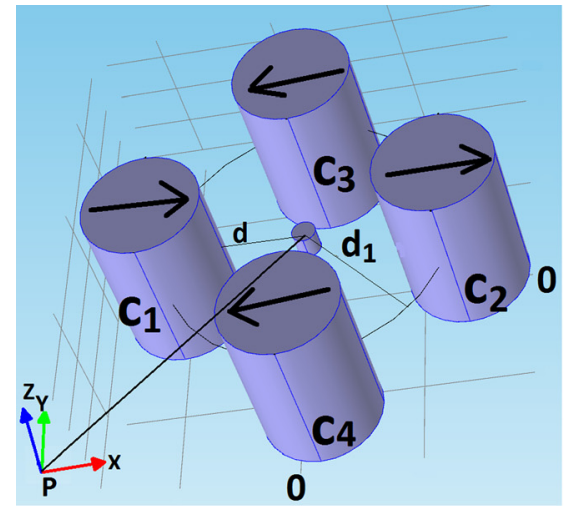

(a)

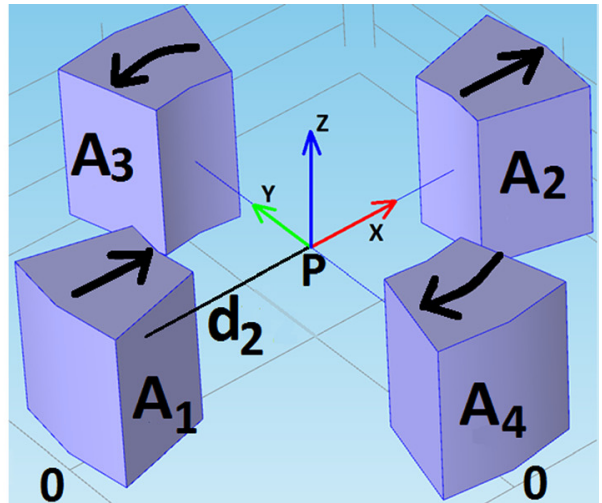

(b)

Fig. 5 (a) Array of four diametrically magnetized cylindrical magnets $C_{i}(i=1,2,3$, and 4) around a small permanent magnet (IPM) whose center is located at point $P$ and $(b)$ four arc-shaped permanent magnets, $A_{i}(i=1,2,3$, and 4$)$, radially and tangentially magnetized. Distance $d_{2}$ is determined from point $\boldsymbol{P}$ to the center of any $\boldsymbol{A}_{\boldsymbol{i}}$. $\boldsymbol{P}$ is located at the center of a circle with a radius $d_{1}$.

with a scaled down structure that consists of four arc-shaped permanent magnets arranged in the configuration denoted as $A_{1234}$.

3.3 Scaled Down External Magnetic Systems. For practical reasons, we work in this section with an operating distance $d$ of $30 \mathrm{~mm}$ and conduct the inverse analysis: we initially estimate $B_{x}$ at point $P$ generated by a given total volume $V_{\text {total }}$ that is equally divided into four arc-shaped permanent magnets (i.e., $A_{1234}$ ). This flux density value is then compared with $B_{x}$ created by the array $C_{1234}$ and also by a single cylindrical EPM. The combined volume of the whole array $C_{1234}$ equals $V_{\text {total }}$ and the volume of the single cylindrical EPM is also $V_{\text {total }}$ and the latter is placed at the position and orientation shown in Figs. 4(a) and 4(b).

The operating distance $d$ of $30 \mathrm{~mm}$, which represents an operating distance of $240 \mathrm{~mm}$ decreased by eight times, is chosen due to the commercial availability of inexpensive arc-shaped permanents magnets with such dimensions that are used in our experimental section. The specifications of these permanent magnets are as follows: magnetization grade of $1.32 \mathrm{~T}$ (i.e., $\mathrm{N} 45$ ), $L_{2}=30 \mathrm{~mm}$, $\Delta \theta=\pi / 6, r_{2}=50 \mathrm{~mm}, r_{1}=30 \mathrm{~mm}, V_{\mathrm{A}}=12.564 \times 10^{-6} \mathrm{~m}^{3}$, and $V_{\text {total }}=4 \times V_{\mathrm{A}}$. The same total volume $V_{\text {total }}$ can be equally divided into four cylindrical EPMs (each cylinder with $R=11.55 \mathrm{~mm}$ and $L_{1}=L_{2}$ ) the centers of which are located at a radial distance $d_{1}$ of $41.55 \mathrm{~mm}$. These two arrays of magnets can be compared in terms of the $B_{x}$ generated at point $P$ with a single cylindrical EPM (with a total volume $V_{\text {total }}, R=23.1 \mathrm{~mm}$, and $L=L_{2}$ ) the center of which is located at the radial distance of $d+R=53.1 \mathrm{~mm}$. Figure 7 shows the comparison of $B_{x}$ along the $x$-axis generated by these scaled down external magnetic systems.

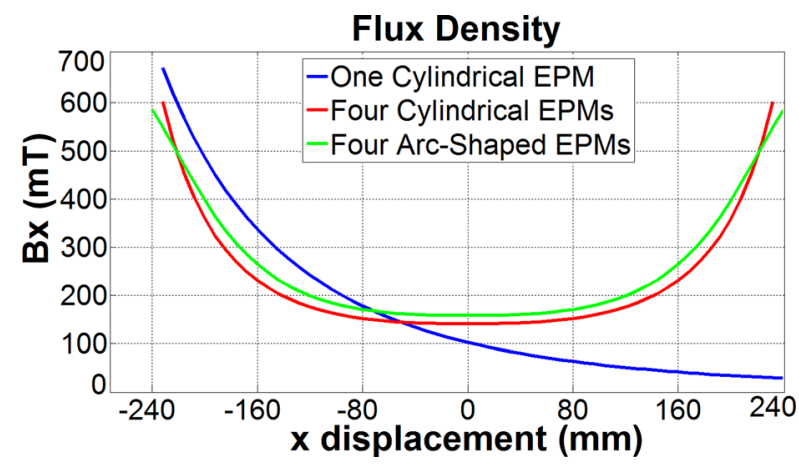

Fig. 6 Comparison of the flux density along the $x$-axis produced by an optimized cylindrical EPM, four cylindrical EPMs, and four arc-shaped magnets. Operating distance $d$ of $240 \mathrm{~mm}$.
The system $A_{1234}$ generates a $B_{x}$ of $113 \mathrm{mT}$ at point $P$, while the structure $C_{1234}$ generates $103 \mathrm{mT}$ and the single cylindrical EPM only generates $74 \mathrm{mT}$. The volume of the single cylindrical EPM would have to be increased to generate $103 \mathrm{mT}$ at point $P$.

The comparisons of $B_{x}$ generated by these external magnetic systems, as shown in Figs. 6 and 7 , indicate that the structure $A_{1234}$ generates the highest $B_{x}$ at point $P$ where the IPM is to be placed. If the IPM is moved along the $x$-axis, it may be subjected to higher flux densities and consequently a higher magnetic torque may be transmitted to the IPM. For example, for some negative values of $x$, a single EPM produces a higher $B_{x}$ but for positive values of $x$, the array $A_{1234}$ can produce higher flux densities. The arrays of magnets also produce a relatively constant value of $B_{x}$ over a larger region along the $x$-axis when compared with the $B_{x}$ produced by a single EPM, thus guaranteeing a more steady torque on the IPM.

With reference to the results in Fig. 7, the flux density generated by the array $A_{1234}$ is considerably higher than the one generated by the array $C_{1234}$ in some regions. For example, the maximum difference in the magnetic flux density between the two arrays is reached at $x=-24 \mathrm{~mm}$ and the difference is $44 \mathrm{mT}$. This is a significant amount that would allow the actuation of small magnets if we consider that a magnitude of $103 \mathrm{mT}$ is used in Ref. [13] to actuate two small magnets. However, in our region of interest where the flux density is minimum (i.e., at $x=0 \mathrm{~mm}$ ), this difference in magnetic flux density is reduced to $10 \mathrm{mT}$, as shown in Fig. 7. Due to this small difference, we believe that either an array of four cylinders or four arc-shaped magnets can be used to improve the magnetic flux density at the center of the system and actuate a small IPM. Nevertheless, since the IPM can be located in other regions along the $x$-axis, we choose to use arc-shaped permanent magnets.

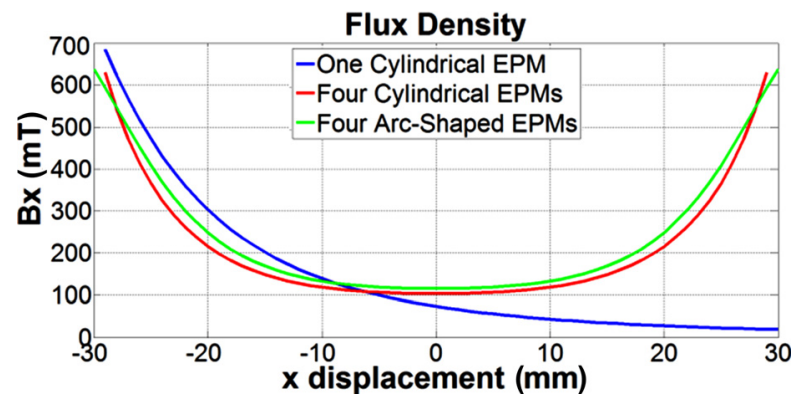

Fig. 7 Comparison of $B_{X}$ produced by a single cylindrical EPM, structure $C_{1234}$, and structure $A_{1234}$ 


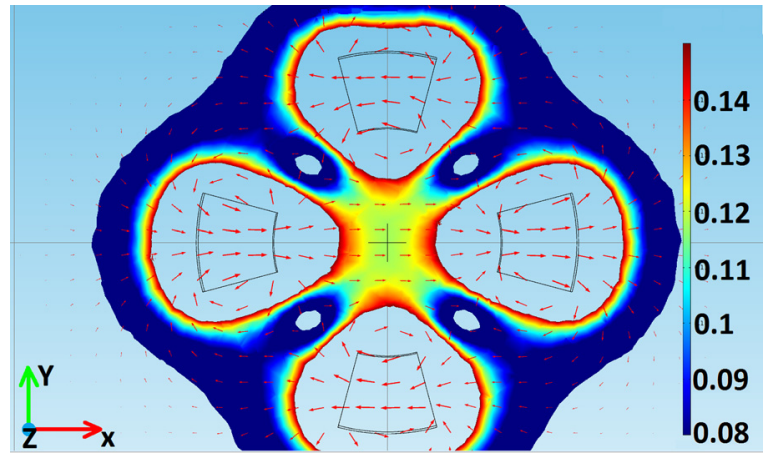

Fig. 8 Vector field of the magnetic flux density norm on the plane $z=0$ generated by the structure $A_{1234}$, when the operating distance $d$ is $30 \mathrm{~mm}$. Scale on the right-hand side is given in Tesla.

The analytical results show that $B_{y}$ is $0 \mathrm{mT}$ along the $x$-axis for both the types of arrays of magnets. For this reason, we do not show $B_{y}$ in any results. However, we present in Fig. 8 a 2D vector field representation of $\left|\mathbf{B}_{\text {ext }}(\mathbf{x})\right|$ created by four arc-shaped magnets in plane $z=0$ (using Comsol).

Figure 8 shows that $\left|\mathbf{B}_{\text {ext }}(\mathbf{x})\right|$ approximates $\mathrm{B}_{\mathrm{x}}$ over a relatively large region around point $P$. Therefore, the $B_{x}$ component is mainly responsible for the transmitted torque on the IPM in Eq. (1).

3.4 Scaling up the Magnetic Systems and Practical Considerations. Although, in Sec. 3.3 we carry out the analytical analysis and comparison of arrays of magnets with an operating distance $d$ of $30 \mathrm{~mm}$, we can scale up the EPMs to increase the operating distance. For instance, in our previous work [16], we use a more realistic operating distance of $240 \mathrm{~mm}$ which represents the scaled down distance multiplied by a factor of 8 . Therefore, we can increase the dimensions of the EPMs presented in Sec. 3.3 proportionally, by using the scaling factor of 8 as follows: for each arc-shaped magnet, we make $L_{2}=30 \times 8=240 \mathrm{~mm}$, $\Delta \theta=\pi / 6, \quad r_{2}=50 \times 8=400 \mathrm{~mm}, \quad$ and $\quad r_{1}=30 \times 8=240 \mathrm{~mm}$ as shown in Fig. 9 $(a)$. For each cylindrical EPM, we make $L_{1}=L_{2}, R=11.55 \times 8=92.4 \mathrm{~mm}$ and its center is located at $d_{1}=41.55 \times 8=332.4 \mathrm{~mm}$ from the center of the system. An arcshaped magnet with such dimensions could be customized by a manufacturer. However, it may be more practical to assemble cheaper and smaller arc-shaped magnets to obtain the same results produced by a single custom arc-shaped magnet as shown in Fig. 9(a) $[27,28]$.
The comparison of the $B_{x}$ produced by these arrays of permanent magnets along the $x$-axis is shown in Fig. $9(b)$.

With reference to Fig. $9(b)$, we find that the maximum difference in the magnetic flux density between the two arrays is reached at $x=-192 \mathrm{~mm}$ (i.e., $x=-24 \mathrm{~mm} \times 8$ or, in other words, this is eight times the value found for the scaled down system) and the difference is $44 \mathrm{mT}$ which is the same value found for the scaled down system. Figures 7-9(b) show that the flux density produced by the array of arc-shaped magnets is always higher than $113 \mathrm{mT}$ and it reaches its minimum value at the center of the system. Considering that the magnetic torque is proportional to the flux density as expressed in Eq. (1), we argue that, with the array $A_{1234}$, a minimum magnetic torque is exerted on the IPM when it is located at the center of the system. If the IPM is located at positions other than the center of the system, as will happen most of the time in the real application of DDS for WCE, a higher magnetic torque can be imparted to the IPM by the array $A_{1234}$ than by using the array of cylindrical EPMs. This is due to the better use of the restricted space in the circle with a radius of $d$ made by certain shapes, such as arc-shaped magnets when compared with cylindrical EPMs or cubic EPMs [28].

Since the center of the system is the critical point to obtain an improved flux density and, consequently, a useful magnetic torque, as it represents the longest distance to the EPMs, we conduct our experiments by placing the IPM at the center of the system. In regard to the orientation of the IPM in a DDS for WCE, it is expected that this will continuously change as the robotic capsule travels through the digestive system. Therefore, its magnetization vector will change direction and may affect the magnetic torque as predicted by Eq. (1). However, the assessment of changes in the magnetic torque due to variations in the IPM's location and orientation to determine the limitations of the system are beyond the scope of this paper.

It is also envisaged that a DDS for WCE will work simultaneously with additional modules, such as an active locomotion system, an anchoring mechanism, and a localization and orientation module. All these modules must be compatible with the magnetic DDS. The active locomotion system would allow the physician to take the robotic capsule to the region of interest by controlling the capsule's position remotely. For example, Ref. [11] presents a torque-driven magnetic system for active locomotion that may be compatible with our proposed magnetic DDS. Once the WCE reaches the target area, the anchoring mechanism would allow the physician to stabilize the robotic capsule before releasing the drug compounds. In this way, the robotic capsule will be able to resist the peristaltic force in the gastrointestinal tract, the magnetic force generated by the EPMs, and the reaction forces generated within the robotic capsule while the drug is being released. A forcedriven magnetic system that allows the capsule to anchor is

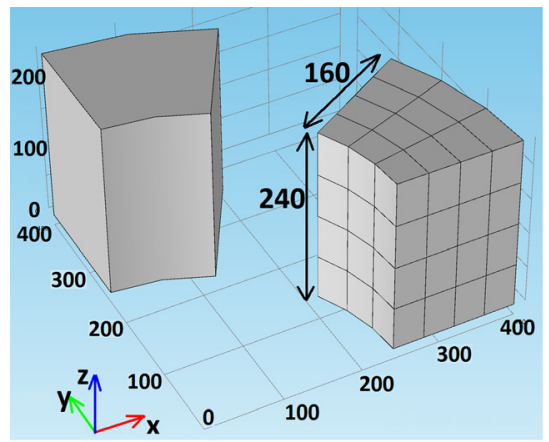

(a)

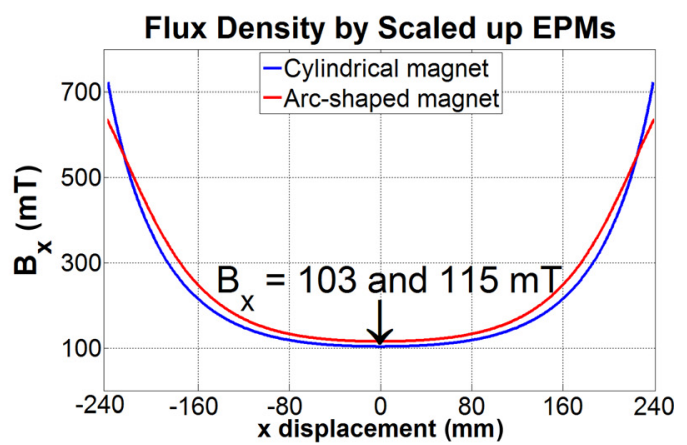

(b)

Fig. 9 (a) Scaled-up magnetic system (dimensions in millimeter). Left: a single custom arcshaped permanent magnet and right: assembly with smaller arc-shaped magnets. (b) Comparison of $B_{x}$ produced by the array of cylindrical magnets (denoted as $C_{1234}$ ) and the array of arc-shaped magnets (denoted as $A_{1234}$ ) when the operating distance $d$ is $240 \mathrm{~mm}$. 


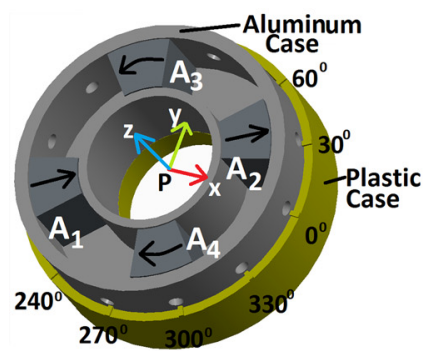

(a)

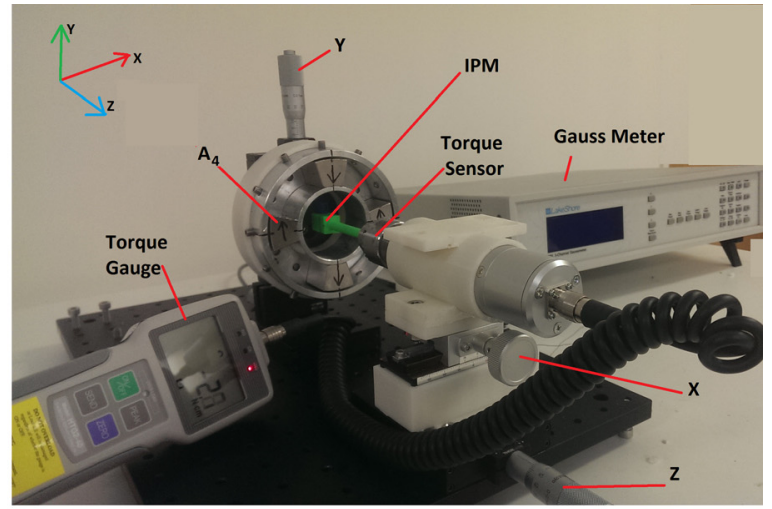

(b)

Fig. 10 (a) EPMs fixed on the aluminum case and rotated by $\theta_{\mathrm{a}}=30 \mathrm{deg}$ and $(b)$ experimental setup consisting of the measurement instruments and the array of arc-shaped permanent magnets

presented in Ref. [3] and it can be compatible with our proposed DDS. Finally, a localization and orientation module embedded in the robotic capsule, such as the localization system based on positron emission markers presented in Ref. [29] which is compatible with our magnetic DDS, would provide information to adjust the $\mathrm{EPMs}^{\prime}$ position and orientation and compensate for misalignments with the IPM if needed. The incorporation of all these additional modules in the restricted volume of a WCE emphasizes again the necessity of miniaturizing the IPM and optimizing the external magnetic system to achieve efficient magnetic linkage at longer operating distances.

For practical reasons, we have decided to experiment with the scaled down magnetic system made of an array of arc-shaped permanent magnets (i.e., structure $A_{1234}$ ) and the details are presented in Secs. 4 and 5.

\section{Experimental Setup for Magnetic Interactions}

The general coordinate system $X Y Z$, detailed in Sec. 2.1, is associated with the fabricated plastic case shown in Fig. 10(a), which possesses $30 \mathrm{deg}$ angle indicators and allows the manual rotation of the array of arc-shaped magnets.

The angle $\theta_{\mathrm{a}}$ represents the misalignment angle between the $X$ and $X_{\mathrm{a}}$ axes as defined in Sec. 2. Figure 10(a) shows, for example, the EPMs rotated by an angle $\theta_{\mathrm{a}}$ of $30 \mathrm{deg}$. In the experimental analysis of the magnetic flux density created by the external magnetic system, we align $X_{\mathrm{a}}$ and $X$ axes (i.e., $\theta_{\mathrm{a}}=0 \mathrm{deg}$ ) because there is no need to rotate the external magnetic system. However, in the experimental analysis of the magnetic torque imparted to the IPM, $\theta_{\mathrm{a}}$ takes values from $0 \mathrm{deg}$ to $360 \mathrm{deg}$ allowing the manual rotation of the external magnetic system.

A three-channel Gauss meter (Lakeshore-Model 460) was used to measure the magnetic flux density generated by the arc-shaped magnets whose dimensions and magnetization grade are defined in Sec. 3.3. A torque gauge (HTG2-40 supplied by IMADA) with its respective torque sensor held the IPM at the center of the system. The IPM was connected to the torque sensor via a plastic connector that was manufactured using a $3 \mathrm{D}$ printer. The torque sensor and the probe tip of the Gauss meter were mounted on plastic holders which were also fabricated using a 3D printer. Both the torque sensor and the probe tip of the Gauss meter can be moved along the $X$ and $Z$ axes and the arrays of magnets can only be moved along the $Y$-axis. These displacements are controlled by a micromanipulation system based on an $X-Y-Z$ stage, as shown in Fig. 10(b). The experimental setup shown in Fig. 10(b) was used to validate the theoretical results for $B_{x}$ generated by the array of arc-shaped magnets and the transmitted magnetic torque on an IPM as it is presented in Secs. 5.1 and 5.2.

\section{Experimental Results With a Prototype of DDS}

In the first series of experiments, we measured the magnetic flux density $B_{x}$ produced by the individual segments $A_{1}$ and $A_{3}$. In the subsequent experiments, we experimentally evaluated the effect of having multiple segments; thus, we measured $B_{x}$ produced by $A_{1}$ and $A_{2}$ acting simultaneously, which we designate as $A_{12}$. Similarly, we measured $B_{x}$ produced by $A_{34}$ and $A_{1234}$, which was the contribution of all the segments acting simultaneously. In all these experiments, the magnets are fixed in their respective positions (i.e., the $Y$-axis does not move). The $z$-position of the probe tip is adjusted until it reaches $z=0$ and then the probe tip is moved from $-21 \mathrm{~mm}$ to $24 \mathrm{~mm}$ along the $x$-axis. All these experimental results were compared with the analytical results and are presented in Sec. 5.1.

5.1 Experimental Results for the Magnetic Flux Density. We can see that the magnetic flux density is enhanced when multiple magnets are added in the system. The maximum $B_{x}$ measured at the center of the system is $114.4 \mathrm{mT}$ with an array of four arcshaped magnets as shown in Fig. 11(c).

5.2 Experimental Results for Magnetic Torques. In the second series of experiments, we were interested in measuring the magnetic torque $\tau_{z}$ exerted on the $6.35 \mathrm{~mm}$ cubic IPM with the magnetization of $1.25 \mathrm{~T}$ (N40) only by segment $A_{1}$. These dimensions and magnetization grade of the IPM are specific in Sec. 5.2, but we vary these parameters in Sec. 5.3 to determine the smallest IPM to be embedded in the robotic capsule. Even though for the same volume and magnetization grade, cylindrical IPMs can produce higher magnetic torques than cubic IPMs [30], we decided to conduct our experiments with the worst scenario (i.e., with cubic IPMs). In the subsequent experiments, we verified the effect of having multiple segments; thus, we measured $\tau_{z}$ produced by $A_{12}$, $A_{123}$, and $A_{1234}$. In all these experiments, the IPM is fixed at the center of the system (i.e., $X=Y=Z=0$ ) and its magnetization vector $\mathbf{m}$ was aligned with the $x$-axis at all times. The arc-shaped magnets rotated about the $z$-axis with increments of $30 \mathrm{deg}$, and therefore, $\varnothing_{\mathrm{m}}$ in Eq. (1) always equals $\theta_{\mathrm{a}}$. The comparison between the analytical results, which are estimated using Eq. (1), and the experimental results for the transmitted magnetic torque $\tau_{z}$ is presented in Fig. 12 .

We can see that the combination of multiple magnets not only improves the magnetic field at the center of the system but also the peak torque on the IPM. The maximum torque exerted by the array $A_{1234}$ on the cubic IPM was measured as $26 \mathrm{mN} \cdot \mathrm{m}$. Although the assessment of the magnetic torque for different 

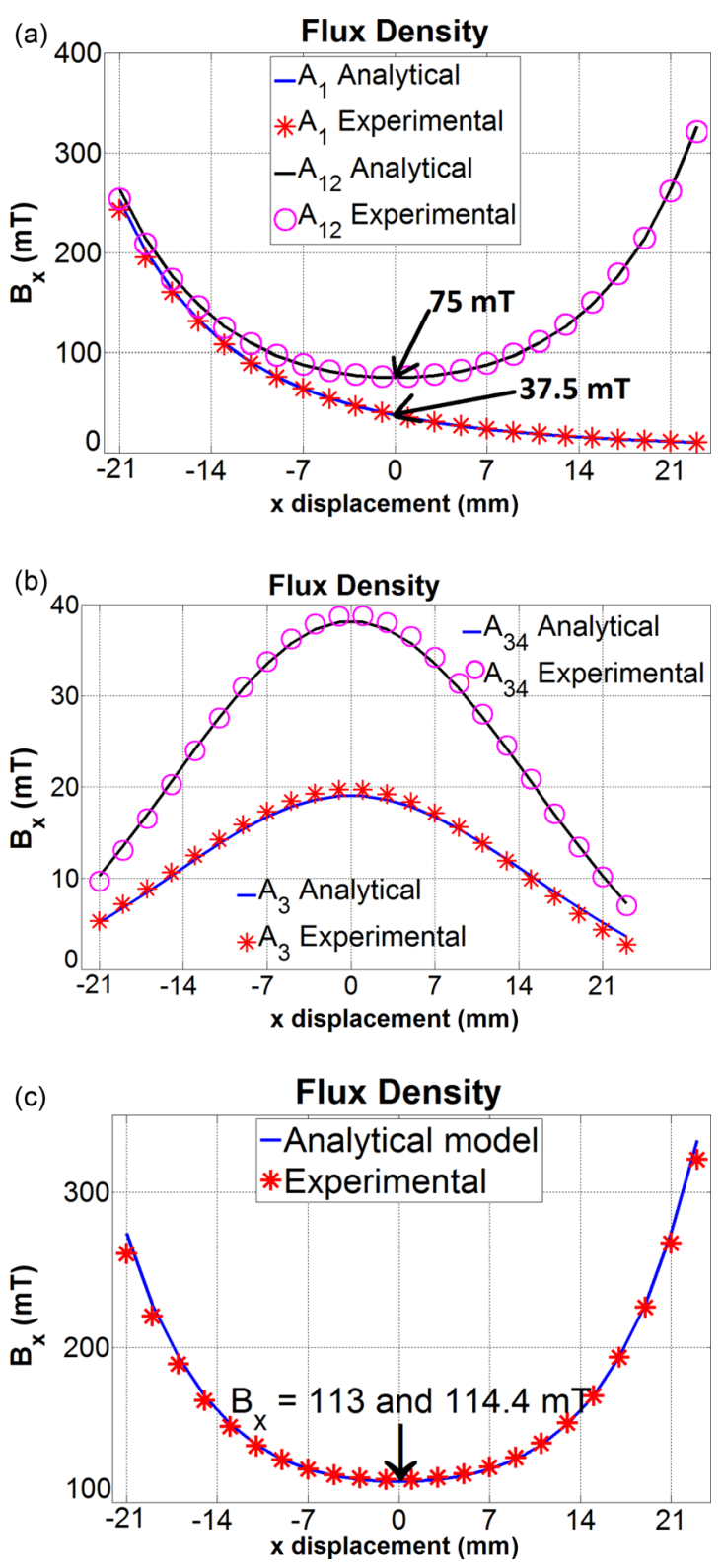

Fig. $11 B_{x}$ produced by arc-shaped magnets: (a) radially magnetized $\left(A_{1}\right.$ and $\left.A_{12}\right),(b)$ tangentially magnetized $\left(A_{3}\right.$ and $\left.A_{34}\right)$, and $(c)$ the array $A_{1234}$

IPM's positions and orientations is not within the scope of this paper, we do present some experimental results in Ref. [30] for an IPM, the center of which is not located at the center of the system. We use the array $A_{1234}$ as the source of the rotating magnetic field to actuate a slider-crank mechanism and the details of this mechanism and the experimental results for it are presented in Sec. 5.3.

5.3 Experimental Results for Piston Force. We connected the cubic IPM to a slider-crank mechanism to convert the rotational motion of the IPM into the translational motion of a piston. The IPM is inserted in its case and its magnetization vector, $\mathbf{m}$, is always parallel to the vector that is projected on the plane $z=0$ and the tail and tip of which are located at the center of the crankshaft and the center of the crankpin, respectively, as shown in Fig. 13(a).

The IPM case can house the cubic magnets ranging from 3.175 to $10 \mathrm{~mm}$. Figure $13(b)$ shows the components of the slider-crank mechanism (i.e., the gray disk, the connecting rod (B), and the

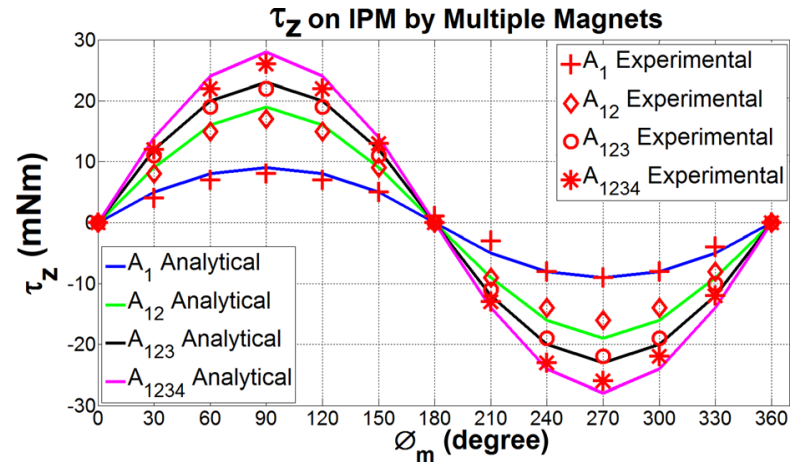

Fig. $12 \tau_{\mathrm{z}}$ produced by single and multiple permanent magnets on the cubic IPM

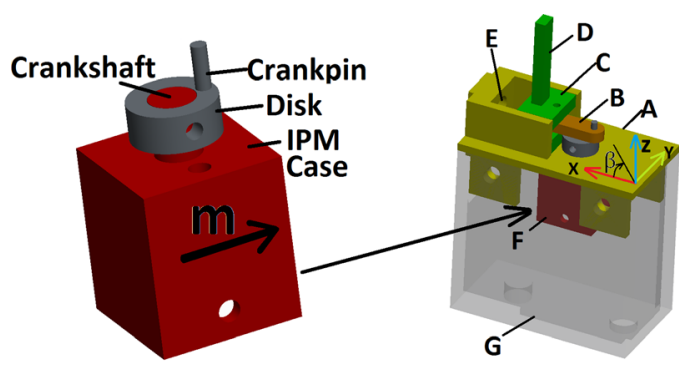

(a)

(b)

Fig. 13 (a) The cubic IPM case connected to a disk through the crankshaft. (b) Components of the slider-crank mechanism. A: Platform, B: connecting rod, C: piston, D: the reflective surface for laser-based displacement measurements, E: spring holder, F: IPM case, G: platform support. $\beta$ is the angle formed by the external magnetic system and the $x$-axis (i.e., $\beta=180$ deg $-\theta_{a}$ and $\theta_{\mathrm{a}}$ is shown in Fig. 10(a)).

green piston $(\mathrm{C})$ shown in Fig. 13(b)), which were fabricated in plastic material with a $3 \mathrm{D}$ printer. The IPM is inserted into the IPM case which is held by the yellow platform (A) that is fixed and attached to the platform support $(\mathrm{G})$. The IPM freely rotates about the crankshaft the center of which is aligned with the center of the external magnetic system. The piston moves back and forth on the surface of the platform (A) that was smoothed to minimize the friction force. Thus, the piston freely moves along the $x$-axis when the IPM is rotated about the $z$-axis by the magnetic torque, $\tau_{z}$, defined in Eq. (1). The coordinate system used throughout Sec. 5.3 is shown in Fig. 13(b).

The platform support, $\mathrm{G}$, is anchored and holds the slider-crank in place.

Referring to Fig. $14, \alpha, R$, and $L$ designate the crank angle, the lengths of the crank, and the connecting rod, respectively. It should be noted that since the IPM is physically connected to the crankshaft, the angle of $\mathbf{m}$ equals the crankshaft angle $\alpha$. $\beta$ is the angle formed by the external magnetic system and the $x$-axis ranges between $-180 \mathrm{deg}$ and $180 \mathrm{deg}$ (see Fig. 13(b)).

The position of the wrist pin (i.e., point B), $x$, and the approximate crankshaft torque, $\tau$, are expressed as follows [31]:

$$
\begin{gathered}
x=R \cos \alpha+L \sqrt{1-\left[\frac{R * \sin \alpha}{L}\right]^{2}} \\
\tau=F R \sin \alpha\left(1+\frac{R}{L} \cos \alpha\right)
\end{gathered}
$$

By using the law of cosines, we can express the crank angle as a function of $x$ as 


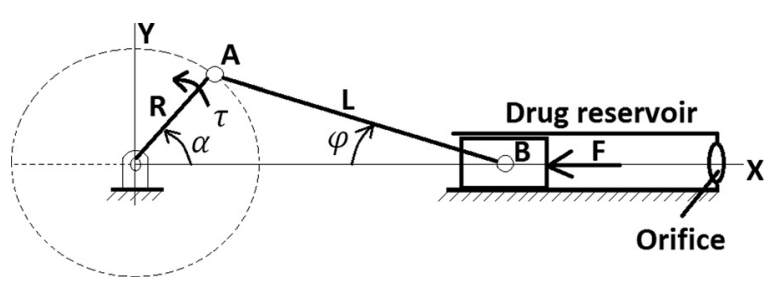

Fig. 14 Slider-crank mechanism for the drug delivery. The $z$-axis is positive into the page.

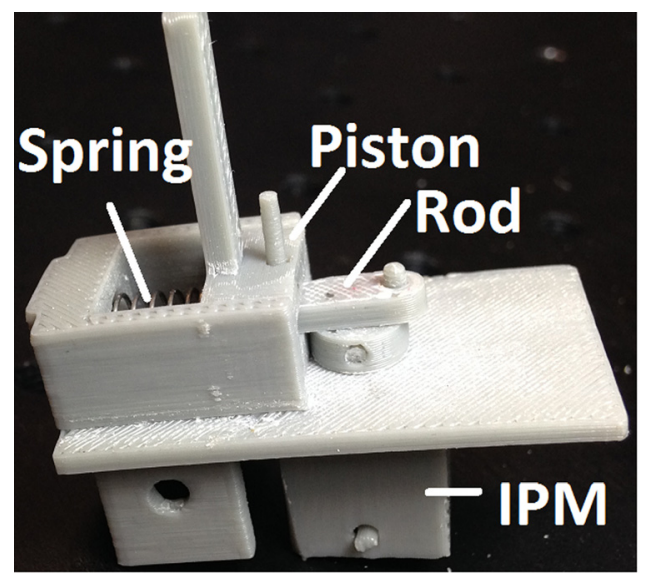

Fig. 15 Components of the slider-crank mechanism and the mechanical spring to measure the piston force

$$
\alpha=\cos ^{-1}\left(\frac{R^{2}+x^{2}-L^{2}}{2 R x}\right)
$$

In order to measure the force delivered to the piston when the IPM rotates, we used a helical spring that is compressed as the piston moves forward and creates the force $F$. The spring is extended when the piston is moved back to its original position, as shown in Fig. 15.

The slider-crank mechanism was fabricated with $R=3 \mathrm{~mm}$ and $L=9 \mathrm{~mm}$, which are dimensions compatible with the size of a commercial WCE. Using these dimensions and Eq. (5), we obtain $x_{\min }=6 \mathrm{~mm}$ and $x_{\max }=12 \mathrm{~mm}$. The force $F$ can be estimated by using the Hooke's law

$$
F=K \Delta x=K\left(x-x_{\min }\right)
$$

where $\Delta x$ represents the displacement of the spring and $K$ is the stiffness of the spring. $K$ was measured as $1.59(\mathrm{~N} / \mathrm{mm})$. In our experiments, we manually rotated the arc-shaped magnets and the cubic IPM rotated at the same time, compressing the spring when $\beta$ changed from $180 \mathrm{deg}$ to $0 \mathrm{deg}$ and extending it when $\beta$ changed from $0 \mathrm{deg}$ to $-180 \mathrm{deg}$. A laser (optoNCDT 1700 by MicroEpsilon) was used to measure $x$ (see Fig. 16(b)). The beam of the laser was targeted on the reflective surface that was connected to the piston, as shown in Fig. 13(b). The laser reading was used to estimate the crank angle $\alpha$ and the spring force $F$, which are both dependent on $x$, as expressed in Eqs. (7) and (8), respectively. Once $F$ and $\alpha$ are estimated, we use Eq. (6) to estimate the torque needed to balance the force $F$.

Figure 16(a) shows the entire system at the initial position. At this position, the magnetization vectors of the segment $A_{2}$ and the cubic IPM are pointing toward $\beta=180 \mathrm{deg}$ and the position of the wrist pin (i.e., point $B$ ) is $6 \mathrm{~mm}$ away from the center of the crank.

We conducted experiments with a variety of cubic IPMs to assess the capability of the system to convert the magnetic torque into a piston force. Table 1 shows the specifications of different

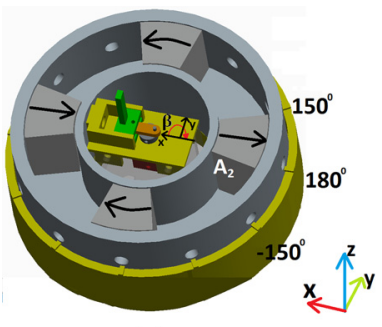

(a)

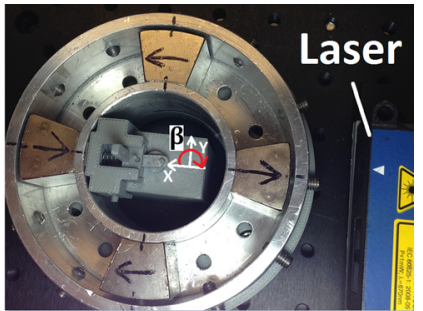

(b)
Fig. 16 (a) External magnetic system powering the slider-crank mechanism and rotated by $\beta=180 \mathrm{deg}$. (b) The laser was used to measure the piston displacement along the $x$-axis.

Table 1 Specifications of IPMs used in the experiments

\begin{tabular}{lcc}
\hline \hline Magnetization grade & $|\boldsymbol{m}|(\mathrm{T})$ & Size $(\mathrm{mm})$ \\
\hline N50 & 1.40 & 3.175 \\
N50 & 1.40 & 4 \\
N40 & 1.25 & 5 \\
N40 & 1.25 & 6.35 \\
N40 & 1.25 & 10 \\
\hline \hline
\end{tabular}

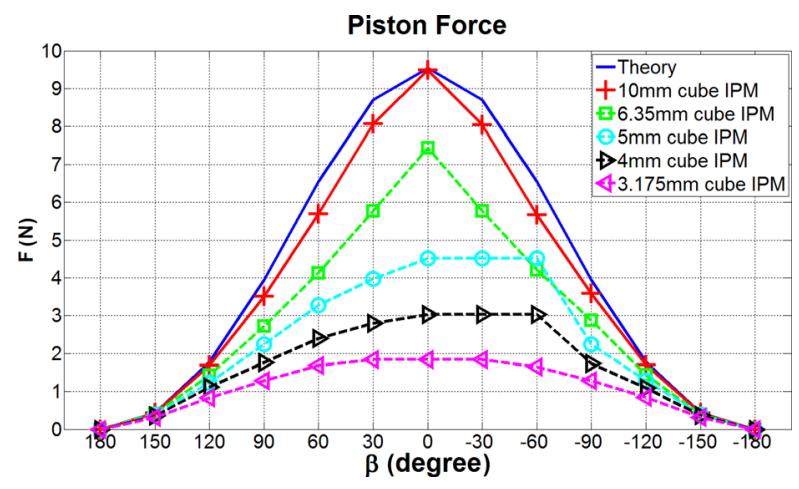

Fig. 17 Piston force response with a variety of cubic IPMs. It shows the compression and extension of the spring in the entire cycle.

IPMs and Fig. 17 shows the spring force $F$ which equals the magnitude of the piston force but its direction is opposite to the piston force direction, thus stabilizing the piston.

Figure 17 shows that, for IPMs smaller than $6.35 \mathrm{~mm}$, the spring reaches a maximum compression at which the piston exerts its peak force. Although peak forces are not required to release the drug from the reservoir, once the piston force reaches its peak value, the piston will not move forward beyond this point. For instance, for the smallest IPM $(3.175 \mathrm{~mm})$, the peak force is obtained when the EPMs are rotated by $\beta=30 \mathrm{deg}$, while this peak is reached at $\beta=0 \mathrm{deg}$ for IPMs of $4 \mathrm{~mm}$ and $5 \mathrm{~mm}$. At the point when the IPMs cannot further compress the spring, the EPMs provide the maximum magnetic torque. However, if we continue rotating the EPMs until they reach approximately $\beta=-90 \mathrm{deg}$ for IPMs of 4 and $5 \mathrm{~mm}$, the spring is extended (i.e., released) abruptly.

Figure 18 shows the crankshaft torque $\tau$ estimated using Eqs. (6)-(8). For instance, when the EPMs were manually rotated until they reached $\beta=60 \mathrm{deg}$, position $x$ was measured as $7.05 \mathrm{~mm}$ for the smallest IPM $(3.175 \mathrm{~mm})$. The force on the piston is estimated to be $F=K \Delta x=1.59 *(7.05-6)=1.67 \mathrm{~N}$ as shown in Fig. 17. Since only position $x$ is measured with the laser sensor, we can estimate crank angle $\alpha$ by using Eq. (7) and we 


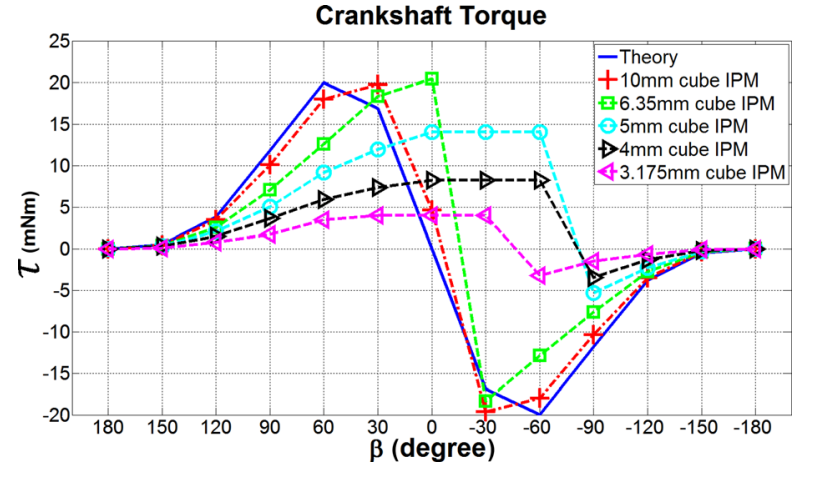

Fig. 18 Crankshaft torque response with a variety of cube IPMs. It shows the compression and extension of the spring in the entire cycle.

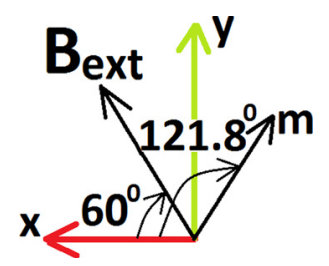

Fig. 19 Vector representation when the EPMs are oriented at $\beta=60$ deg. This vector representation is a top view of the coordinate system defined in Fig. 13(b).

found $\alpha$ to be $121.81 \mathrm{deg}$. We then used $\alpha$ and force $F$ in Eq. (8) to estimate crankshaft torque $\tau$ of $3.51 \mathrm{mN} \cdot \mathrm{m}$ as shown in Fig. 18 .

This torque $\tau$ exerted by the spring on the crankshaft should be equal in magnitude (but in the opposite direction) to the magnetic torque $\tau_{z}$ produced by the EPMs acting on the IPM. Thus, we can use Eq. (1) to validate the result obtained for the crankshaft torque. When the EPMs are rotated by $\beta=60 \mathrm{deg}$, the crank angle $\alpha$ was calculated as $121.81 \mathrm{deg}$ with respect to the $x$-axis. Since the IPM is physically connected to the crankshaft, the magnetization vector $\mathbf{m}$ forms an angle of $121.81 \mathrm{deg}$ with respect to the $x$-axis, as shown in Fig. 19.

We use the following values in Eq. (1): $V=3.2 \times 10^{-8} \mathrm{~m}^{3}$ (for the smallest IPM), magnetization of $1.4 \mathrm{~T},\left|\mathbf{B}_{\text {ext }}\right|$ was measured as $114.4 \mathrm{mT}$ at the center of the system (see Fig. 11(c)) and $\varnothing_{\mathrm{m}}=60 \mathrm{deg}-121.81 \mathrm{deg}=-61.81 \mathrm{deg}$ and we estimate $\tau_{\mathrm{z}}$ to be $-3.59 \mathrm{mN} \cdot \mathrm{m}$. The negative value of the magnetic torque $\tau_{\mathrm{z}}$ indicates that this torque is in the clockwise direction; thus, it approximately balances the crankshaft torque $\tau$ of $3.51 \mathrm{mN} \cdot \mathrm{m}$ in the counter clockwise direction shown in Fig. 14.

There is only a small difference between the magnitudes of $\tau_{\mathrm{z}}$ and $\tau$ (less than $0.1 \mathrm{mN} \cdot \mathrm{m}$ ), thus validating our results. We postulate that this small difference could be due to imperfections in the experimental setup, such as friction force and clearances at the joints. In order to overcome the limitations associated with the accuracy of $3 \mathrm{D}$ printing, all the components of the slider-crank mechanism can be fabricated more precisely using, for example, the LiGA process [32]. The improvement in the fabrication of these components will be useful for the final integration of the mechanism in commercial WCEs.

5.4 Drug Delivery Capability. We fabricated a cubic piston with a cross-sectional area of $105.6 \mathrm{~mm}^{2}(12 \mathrm{~mm} \times 8.8 \mathrm{~mm})$ and a maximum stroke of $6 \mathrm{~mm}\left(X_{\max }-X_{\min }=2 R=6 \mathrm{~mm}\right)$. These dimensions give a total drug reservoir volume of $0.633 \mathrm{ml}$. Compared to the reservoir of the capsule-based DDSs reported in the literature [1,23], which ranges between $0.17 \mathrm{ml}$ and $1 \mathrm{ml}$, this is a reasonable drug reservoir volume. We can fabricate the slider-crank mechanism with a longer stroke or a bigger cross-sectional area to easily bring the drug reservoir volume to $1 \mathrm{ml}$. For example, if we increase the length of the crank to $R=5 \mathrm{~mm}$ and use a cylindrical piston with a cross-sectional area of $95 \mathrm{~mm}^{2}$ (i.e., by considering the typical diameter of a WCE which is $11 \mathrm{~mm}$ [1]), then we obtain a maximum stroke of $2 R=10 \mathrm{~mm}$. These dimensions would result in a total drug reservoir of $0.950 \mathrm{ml}$.

The number of doses that our fully controllable prototype of DDS can release depends on the pharmaceutical or treatment needs. For instance, if the total drug reservoir volume is divided by 6 , with volumes of $0.105 \mathrm{ml}$ each; then, the maximum number of doses to deliver would be 6 . In this case, we can rotate the external magnetic system to make the piston advance by increments of $1 \mathrm{~mm}$ each time. The first increment can be obtained when the crank angle changes from $180 \mathrm{deg}$ to $123 \mathrm{deg}$ and the second drug release requires the crank angle to change from $123 \mathrm{deg}$ to approximately $100 \mathrm{deg}$. The third release would be possible by decreasing the crank angle to $80 \mathrm{deg}$ and we can continue releasing the drug until the crank angle becomes 0 deg in a nonlinear fashion.

If more than six doses are required, the number of doses and release amount can be precisely controlled by making the piston advance in smaller increments as long as the torque load of the drug payload remains under the peak torque value imparted to the IPM. Finally, the release rate will depend on the rotational speed of the external magnetic system. Although in this work we manually rotated the external magnetic system, its rotational speed could be more precisely controlled by using motors and a control station along with a joystick, as we proposed in Sec. 2.

\section{Conclusions and Future Work}

It is highly desirable for an effective and accurate DDS to be included in the next generation of WCE. Several requirements must be fulfilled, however, for the successful development of such a system, and these include the active actuation of an untethered releasing mechanism that allows the control of variables, such as the release rate, release amount, and number of doses. In this paper, we focus on the optimization of an external magnetic system and the dimensions of the IPM to remotely actuate a drug release mechanism for CE.

We investigate the most suitable external magnetic system to produce the highest rotating magnetic field under which a small internal magnet (i.e., IPM) could be used. We compare a single cylindrical permanent magnet against arrays of cylindrical and arc-shaped permanent magnets as the source of the rotating magnetic field. We find that, for the same volume, the arrays of permanent magnets can produce stronger magnetic fields than a single cylindrical magnet. We also find that either cylindrical or arcshaped magnets are appropriate to improve the magnitude of the magnetic field at the center of the system. However, arc-shaped magnets can produce higher magnetic fields in regions where the IPM is also expected to be. Therefore, arc-shaped magnets provide advantages over the cylindrical magnets in reducing the volume and weight of the external magnetic system. Since these advantages are important to reduce the fabrication costs and also ease the maneuverability of the external magnetic system, we used arc-shaped magnets in our experiments and verified that the combination of four magnets (at optimized locations and orientations) not only improved the magnetic field at the center of the system but also the peak torque on the IPMs. Since these results are based on analytical models that are valid for different sizes of magnets, we conclude that an array of multiple arc-shaped magnets can be scaled-up and placed at longer distances from the center of the system to actuate a small IPM embedded in a robotic capsule.

The magnetic flux density generated by four arc-shaped magnets was measured at the center of the system as $114 \mathrm{mT}$. Several cubic IPMs acting independently were used to actuate the piston that would expel drug out of a reservoir. We assessed the 
capability of each cubic IPM to convert the magnetic torque into a piston force and found that the smallest cubic IPM (i.e., $3.175 \mathrm{~mm}$ ) produced a peak piston force of $1.67 \mathrm{~N}$. Considering that a peak piston force of only $820 \mathrm{mN}$ is needed to release a variety of drug compounds [16], we conclude that even the $3.175 \mathrm{~mm}$ cubic IPM is sufficient to release drugs and further miniaturization of the IPM is still possible. Nevertheless, a further miniaturization and compactness of the slider-crank mechanism is also needed to leave sufficient room within the capsule robot to integrate additional modules such as the image guidance and anchoring mechanism to improve the accuracy of the drug release procedure. The $3.175 \mathrm{~mm}$ cubic IPM which we used is the smallest size that has been used in a prototype of robotic capsule (if compared to the ones reported in the literature [3,10-15]). Therefore, our optimized external magnetic system guarantees that an adequate amount of magnetic field is produced to actuate the IPM while providing the following benefits: a longer operating distance, enough volume for the drug reservoir, high control over the number of doses, and the release amount. Furthermore, the optimized magnetic system is able to actuate the drug release module when the capsule is located not only at the center of the system, but also at any other point within the region of operation, which is of great advantage for the irregular transport process of the capsule through the biological tract.

In regard to the experimental results for the crankshaft torque, we have found that a peak torque of about $3.5 \mathrm{mN} \cdot \mathrm{m}$ (which is converted into a peak piston force of $1.67 \mathrm{~N}$ ) is adequate to actuate the piston. In order to generate smooth movements in the piston, however, the magnetic system should be designed in such a way that it is always able to generate a magnetic peak torque that exceeds the crankshaft torque requirement at any angle of orientation of the EPMs. However, the peak force and torque are not always required to release the drug compound. Future experimental quantification is required to determine the capability of the system to release different drug forms including powder and liquid. Our future experiments will evaluate the release rate by considering an analysis at a constant angular velocity rather than rotating the external magnetic system manually. Finally, an anchoring system is needed in the robotic capsule to resist the peristaltic force while the drug is being released in the gastrointestinal tract. This mechanism and a smaller slider-crank mechanism will be included in our future work along with the assessment of torque transmission when there are variations in the IPM's location and orientation.

\section{Acknowledgment}

This work was supported in part by the Intelligent Nano-Tera Research Systems Laboratory. The work of F. Munoz was supported by a Ph.D. scholarship from the University of Wollongong Research Council. The authors wish to gratefully acknowledge the help of Dr. Madeleine Strong Cincotta in the final language editing of this paper.

\section{References}

[1] Munoz, F., Alici, G., and Li, W., 2014, "A Review of Drug Delivery Systems for Capsule Endoscopy,” Adv. Drug Delivery Rev., 71, pp. 77-85.

[2] van der Schaar, P. J., Dijksman, J. F., Broekhuizen de Gast, H., Shimizu, J., van Lelyveld, N., Zou, H., Lordanov, V., Wanke, C., and Siersema, P. D., 2013, “A Novel Ingestible Electronic Drug Delivery and Monitoring Device," Gastrointest. Endoscopy J., 78(3), pp. 520-528.

[3] Yim, S., and Sitti, M., 2012, "Design and Rolling Locomotion of a Magnetically Actuated Soft Capsule Endoscope," IEEE Trans. Rob., 28(1), pp. 183-194.

[4] Abbott, J. J., Peyer, K. E., Lagomarsino, M. C., Zhang, L., Dong, L., Kaliakatsos, I. K., and Nelson, B. J., 2009, "How Should Microrobots Swim?" Int. J. Rob. Res., 28, pp. 1434-1447.

[5] Belharet, K., Folio, D., and Ferreira, A., 2011, "MRI-Based Microrobotic System for the Propulsion and Navigation of Ferromagnetic Microcapsules," Minimally Invasive Ther. Allied Technol., 19(3), pp. 157-169.
[6] Bergeles, C., and Yang, G.-Z., 2014, "From Passive Tool Holders to Microsurgeons: Safer, Smaller, Smarter Surgical Robots," IEEE Trans. Biomed. Eng., 61(5), pp. 1565-1576.

[7] Mahoney, A. W., and Abbott, J. J., 2014, "Generating Rotating Magnetic Fields With a Single Permanent Magnet for Propulsion of Untethered Magnetic Devices in a Lumen," IEEE Trans. Rob., 30(2), pp. 411-420.

[8] Petruska, A. J., and Abbott, J. J., 2014, "Omnimagnet: An Omnidirectional Electromagnet for Controlled Dipole-Field Generation," IEEE Trans. Magn., 50(7), p. 8400810.

[9] Peyer, K. E., Zhangb, L., and Nelson, B. J., 2013, "Bio-Inspired Magnetic Swimming Microrobots for Biomedical Applications," Nanoscale, 5(4) pp. $1259-1272$.

[10] Lien, G. S., Liu, C. W., Jiang, J. A., Chuang, C. L., and Teng, M. T., 2012, "Magnetic Control System Targeted for Capsule Endoscopic Operations in the Stomach-Design, Fabrication, and In Vitro and Ex Vivo Evaluations," IEEE Trans. Biomed. Eng., 59(7), pp. 2068-2079.

[11] Sun, Z.-J., Ye, B., Qiu, Y., Cheng, X.-G., Zhang, H.-H., and Liu, S., 2014, "Preliminary Study of a Legged Capsule Robot Actuated Wirelessly by Magnetic Torque," IEEE Trans. Magn., 50(8), p. 5100706.

[12] Sun, Z-J., Cheng, X-G., Cao, S., Ye, B, Zhang, H.-H, and Liu, S., 2014 "Multi-Applications of a Magnet Configuration in Actuating Capsule Endoscope,” IEEE/ASME International Conference on Advanced Intelligent Mechatronics (AIM), Besacon, France, July 8-11, pp. 106-111.

[13] Simi, M., Gerboni, G., Menciassi, A., and Valdastri, P., 2013, "Magnetic Torsion Spring Mechanism for a Wireless Biopsy Capsule," ASME J. Med. Devices, 7(4), p. 041009.

[14] Yim, S., Gultepe, E., Gracias, D., and Sitti, M., 2013, "Biopsy Using a Magnetic Capsule Endoscope Carrying, Releasing and Retrieving Untethered Micro-Grippers," IEEE Trans. Biomed. Eng., 61(2), pp. 513-521.

[15] Gorlewicz, J. L., Battaglia, S., Smith, B. F., Ciuti, G., Gerding, J., Menciassi, A., Obstein, K. L., Valdastri, P., and Webster, R. J., 2013, "Wireless Insufflation of the Gastrointestinal Tract," IEEE Trans. Biomed. Eng., 60(5), pp. $1225-1233$.

[16] Munoz, F., Alici, G., and Li, W., 2014, "Design Optimization of a Magnetomechanical System for Drug Delivery in Wireless Capsule Endoscopy," IEEE/ASME International Conference on Advanced Intelligent Mechatronics (AIM), Besacon, France, July 8-11, pp. 1097-1102.

[17] Woods, S. P., and Constandinou, T. G., 2013, "Wireless Capsule Endoscope for Targeted Drug Delivery: Mechanics and Design Considerations," IEEE Trans. Biomed. Eng., 60(4), pp. 945-953.

[18] Montague, R. G., Bingham, C., and Atallah, K., 2013, "Magnetic Gear PoleSlip Prevention Using Explicit Model Predictive Control," IEEE/ASME Trans. Mechatron., 18(5), pp. 1535-1543.

[19] Agashe, J. S., and Arnold, D. P., 2008, "A Study of Scaling and Geometry Effects on the Forces Between Cuboidal and Cylindrical Magnets Using Analytical Force Solutions," J. Phys. D, 41(10), pp. 1-9.

[20] Furlani, E. P., 2000, "Analytical Analysis of Magnetically Coupled Multipole Cylinders," J. Phys. D, 33(1), pp. 28-33.

[21] Jørgensen, F. T., Andersen, T. O., and Rasmussen, P. O., 2008, "The Cycloid Permanent Magnetic Gear," IEEE Trans. Ind. Appl., 44(6), pp. 1659-1665.

[22] Furlani, E. P., 1993, "Formulas for the Force and Torque of Axial Couplings," IEEE Trans. Magn., 29(5), pp. 2295-2301.

[23] Furlani, E. P., Reanik, S., and Janson, W., 1994, "A Three-Dimensional Field Solution for Bipolar Cylinders," IEEE Trans. Magn., 30(5), pp. 2916-2919.

[24] Ravaud, R., and Lemarquand, G., 2010, "Magnetic Field Created by Uniformly Magnetized Tile Permanent Magnet," Prog. Electromagn. Res. B, 24, pp. 17-32.

[25] Munoz, F., Alici, G., and Li, W., 2015, "Optimization of Multiple Arc-Shaped Magnets for Drug Delivery in a Capsule Robot," IEEE/ASME International Conference on Advanced Intelligent Mechatronics (AIM), Busan, July 7-11, pp. 1-7.

[26] Windt, C. W., Soltner, H., Dusschoten, D. V., and Blümler, P., 2011, “A Portable Halbach Magnet That Can Be Opened and Closed Without Force: The NMR-CUFF," J. Magn. Reson., 208(1), pp. 27-33.

[27] Sakellariou, D., Hugon, C., Guiga, A., Aubert, G., Cazaux, S., and Hardy, P., 2010, "Permanent Magnet Assembly Producing a Strong Tilted Homogeneous Magnetic Field: Towards Magic Angle Field Spinning NMR and MRI," Magn. Reson. Chem., 48(12), pp. 903-908.

[28] Soltner, H., and Blümler, P., 2010, "Dipolar Halbach Magnet Stacks Made From Identically Shaped Permanent Magnets for Magnetic Resonance," Concepts Magn. Reson., Part A, 36A(4), pp. 211-222.

[29] Than, T. D., Alici, G., Harvey, S., O'Keefe, G., Zhou, H., Li, W., Cook, T., and Alam-Fotias, S., 2014, "An Effective Localization Method for Robotic Endoscopic Capsules Using Multiple Positron Emission Markers," IEEE Trans. Rob., 30(5), pp. 1174-1186.

[30] Munoz, F., Alici, G., and Li, W., 2015, "An Accurate Model for Size Optimization of an Embedded Permanent Magnet for Drug Delivery With Capsule Robots," IEEE International Conference on Technologies for Practical Robot Applications (TePRA), Woburn, MA, May 11-12, pp. 1-7.

[31] Shigley, J. E., and Uicker, J. J. J., 1995, Theory of Machines and Mechanisms, McGraw-Hill, Sydney, pp. 616-629.

[32] Park, S., Koo, K., Kim, G. S., Bang, S. M., Park, J. Y., Song, S. Y., and Cho, D. D., 2008, "A Novel Microactuator for Microbiopsy in Capsular Endoscopes," J. Micromech. Microeng., 18(2), pp. 25-32. 